\title{
Multiple states in visual working memory: Evidence from oculomotor capture by memory-matching distractors
}

\author{
Valerie M. Beck ${ }^{1} \cdot$ Timothy J. Vickery ${ }^{1}$ \\ Published online: 29 April 2019 \\ (C) The Psychonomic Society, Inc. 2019
}

\begin{abstract}
Visual working memory (VWM) representations interact with attentional guidance, but there is controversy over whether multiple VWM items simultaneously influence attentional guidance. Extant studies relied on continuous variables like response times, which can obscure capture - especially if VWM representations cycle through interactive and non-interactive states. Previous conflicting findings regarding guidance when under high working memory (WM) load may be due to the use of noisier response time measures that mix capture and non-capture trials. Thus, we employed an oculomotor paradigm to characterize discrete attentional capture events under both high and low VWM load. Participants held one or two colors in memory, then executed a saccade to a target disk. On some trials, a distractor (sometimes VWM-matching) appeared simultaneously with the target. Eye movements were more frequently directed to a VWM-matching than a non-matching distractor for both load conditions. However, oculomotor capture by a VWM-matching distractor occurred less frequently under high compared with low load. These results suggest that attention is automatically guided toward items matching only one of two colors held in memory at a time, suggesting that items in VWM may cycle through attention-guiding and not-guiding states when more than one item is held in VWM and the task does not require that multiple items be maintained in an active, attention-guiding state.
\end{abstract}

Keywords Visual attention · Visual working memory · Eye movements · Attentional guidance

\section{Introduction}

Although attention and visual working memory (VWM) have long been regarded as deeply connected, controversies regarding the nature of this relationship still abound. Some propose that representations in VWM can be held in different states (Olivers, Peters, Houtkamp, \& Roelfsema, 2011), "active" or "accessory," such that an item held in an active state can influence the guidance of attention, but an item held in an accessory state cannot. When a single item is held in VWM, attention is automatically captured by memory-matching items (e.g., Olivers, Meijer, \& Theeuwes, 2006), suggesting that a single item is by default in an active state and influences attentional guidance. When multiple items are held in VWM,

Electronic supplementary material The online version of this article (https://doi.org/10.3758/s13423-019-01608-7) contains supplementary material, which is available to authorized users.

Timothy J. Vickery

tim.vickery@gmail.com

1 Department of Psychological and Brain Sciences, University of Delaware, 108 Wolf Hall, Newark, DE 19716, USA however, it is unclear whether the default state of all items will be active, accessory, or a mixture of the two.

In some studies, multiple VWM items interacted with attentional guidance (Bahle, Beck, \& Hollingworth, 2018; Beck \& Hollingworth, 2017; Beck, Hollingworth, \& Luck, 2012; Grubert \& Eimer, 2015, 2016; Hollingworth \& Beck, 2016), suggesting that multiple VWM items were maintained in an active state, whereas other studies found reduced influence at higher VWM loads (Downing \& Dodds, 2004; Houtkamp \& Roelfsema, 2006; Soto, Greene, Chaudhary, \& Rotshtein, 2012; van Moorselaar, Theeuwes, \& Olivers, 2014), suggesting that multiple VWM items were maintained in an accessory state. However, much of the previous work used paradigms that rely on continuous measures like response times (RTs), potentially obscuring the effects of attentional capture by collapsing across capture and non-capture trials, or paradigms for which maintaining multiple VWM items in an active state was task-relevant, potentially over-representing the interaction between VWM and attentional guidance.

To overcome these hurdles, we used a combined memory and eye-movement task that has previously shown VWMbased modulation of saccade trajectory (Hollingworth, Matsukura, \& Luck, 2013), allowing us to discretely 
categorize attentional capture and non-capture trials. Participants were shown one or two colors to remember, then instructed to saccade to a left or right disk. After fixating the target disk, participants completed a forced-choice memory test for one remembered color. On some trials, a distractor disk appeared above or below central fixation simultaneously with the target disk. This distractor sometimes appeared in a VWM-matching color, letting us examine oculomotor capture. Unlike RTs, oculomotor capture provides a discrete measure - did the first saccade go toward the target or the distractor? This allows a clearer view of how the probability of attentional capture is affected by memory load.

We hypothesized that, when items are maintained in VWM but are not task relevant, they are held in a mixture of active and accessory states such that not more than one of the items is maintained in an active state at any particular time, on average. A distractor matching an item held in an accessory state would be less likely to capture attention whereas a distractor matching an item held in an active state would be more likely to capture attention. This account predicts that capture by memory-matching items should occur under both load-1 and load-2, but the precise rate of capture should be reduced under load- 2 compared to load- 1 because there is now a chance that the memory-matching distractor on load-2 trials does not match the item currently maintained in an active state in VWM. In fact, the rate of capture under load-2 should be reduced by at least half, if only one of the two items was held in an active state at any given time.

\section{Method}

\section{Participants}

Thirty University of Delaware (UD) students who reported having normal color vision participated for course credit or were compensated US $\$ 10 / \mathrm{h}$ for one 1.5-h session. Based on independent pilot data, we determined we needed a minimum of 19 participants to obtain $95 \%$ power to detect the weakest effect present in our pilot data $\left(\eta_{p}^{2}=.48\right)$ at the standard .05 alpha error probability. We recruited until the usable sample included data from 20 participants (age $\mathrm{M}=18.5$ years, $\mathrm{SD}=$ 0.8 , female $=16$ ) based on the exclusion criteria outlined in the study preregistration (see Results for criteria). All procedures were approved by the UD Institutional Review Board.

\section{Stimuli, apparatus, and procedure}

Stimuli were presented on an LCD monitor $(120 \mathrm{~Hz})$ at a $94-$ $\mathrm{cm}$ viewing distance. Eye position was recorded at $1,000 \mathrm{~Hz}$ using an Eyelink1000 eye-tracking system. Saccades were defined using a combined velocity ( $>35 \%$ s) and acceleration $\left(>9,500 \% \mathrm{~s}^{2}\right)$ threshold. Participants completed a 9-point calibration at the beginning of each block (every 80 trials) and mid-block as needed.

Each trial began with a $500-\mathrm{ms}$ presentation of two colored squares, each subtending $1.2^{\circ}$ visual angle, offset diagonally by $1.13^{\circ}$ visual angle from central fixation (see Fig. 1). The memory array squares could be the same color (load-1) or different colors (load-2). After a blank retention interval $(700,800,900$, or $1,000 \mathrm{~ms})$, a target disk $\left(0.86^{\circ}\right.$ visual angle) appeared left or right of fixation (disk was centered at $4.61-7.06^{\circ}$ visual angle from fixation) and was equally likely to match or not match one memory color. On some trials, the left/right target disk appeared simultaneously with a distractor disk $\left(0.94^{\circ} \mathrm{vi}-\right.$ sual angle) located above or below fixation $\left(2.14^{\circ}\right.$ visual angle from fixation) and could also match a memory color. These factors yielded five trial types for each memory load: target match, no distractor $\left(\mathrm{T}_{\mathrm{M}} \mathrm{D}_{\mathrm{X}}\right)$; target nonmatch, no distractor $\left(\mathrm{T}_{\mathrm{N}} \mathrm{D}_{\mathrm{X}}\right)$; target match, distractor non-match $\left(\mathrm{T}_{\mathrm{M}} \mathrm{D}_{\mathrm{N}}\right)$; target non-match, distractor nonmatch $\left(\mathrm{T}_{\mathrm{N}} \mathrm{D}_{\mathrm{N}}\right)$; and target non-match, distractor match $\left(T_{N} D_{M}\right)$. Participants were instructed to saccade to the left/right target disk and ignore up/down distractors. Disks remained on-screen until the participant made an eye movement toward the target ${ }^{1}$ or the saccade task timed out $(2,000 \mathrm{~ms})$. Once the saccade task was completed, two squares $\left(1.2^{\circ}\right.$ visual angle each $)$ appeared on either side of fixation (offset by $2.0^{\circ}$ visual angle) and participants were instructed to indicate via key press which square matched a color in memory (left button for left square match, right button for right square match). One color was from the memory array, and the other was a foil drawn from the same color family.

Object colors were drawn from four color families (reds, greens, blues, or pinks) containing four exemplars each, and objects were presented on a light gray background. On load-2 trials, memory colors were selected from different color families (e.g., one blue, one pink) and colors of any non-matching target or distractor disks were selected from remaining color families (e.g., red, green). When a disk matched a memory color, the matching color could be exact (same exemplar as the memory array) or inexact (different exemplar from the same color family). When the target or distractor was an inexact match, the color exemplar used for the disk reappeared as the foil in the memory test. Thus, there was no incentive to preferentially attend to or fixate disks that matched a color in memory.

Participants first performed 20 practice trials and then six to eight blocks of experimental trials, depending on how many

\footnotetext{
${ }^{1}$ A threshold was set such that trials terminated successfully when the $\mathrm{x}$ coordinate of the eye position was within $0.86^{\circ}$ visual angle of the target's $\mathrm{x}$ coordinate.
} 


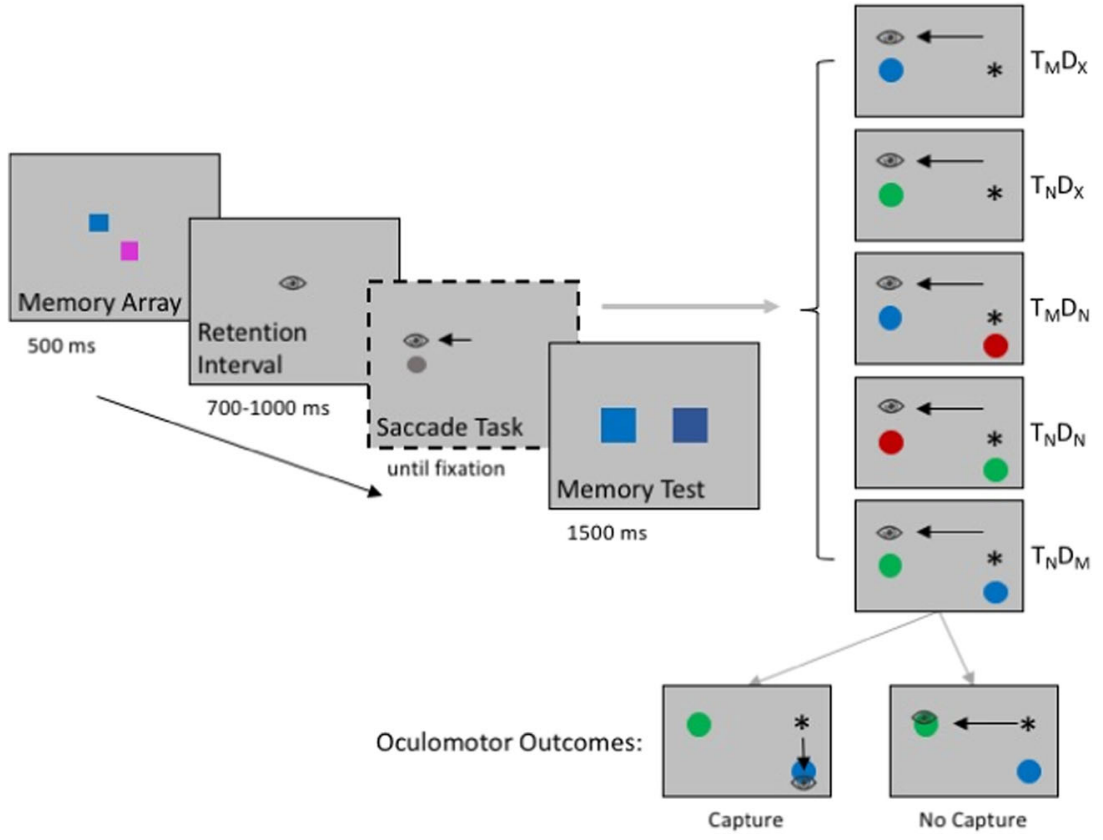

Fig. 1 Illustration of trial events, trial types ( $\mathrm{T}=$ target, $\mathrm{D}=$ distractor, $\mathrm{M}$ = match, $\mathrm{N}=$ non-match, $\mathrm{X}=$ absent), and discrete oculomotor outcomes ("capture," "no capture"). Participants were instructed to remember the memory array color(s), make an eye movement to a left or right target disk, then indicate which of the two colors presented during the memory

were completed during the 1.5 -h session. Each block contained 80 total trials, eight each of the ten trial types, randomly intermixed.

\section{Results and discussion}

One participant was excluded for memory test accuracy no better than chance. Two participants were excluded for executing saccades to a distractor location on $10 \%$ or more of distractor-absent trials, indicative of poor eye tracking or misunderstanding the task. Lastly, seven participants who had $20 \%$ or greater trials excluded from one or more conditions due to the following criteria were excluded from analysis: Initial saccade faster than $90 \mathrm{~ms}$ or slower than $600 \mathrm{~ms}$, initial saccade toward an irrelevant location, or missing eyemovement data. Exclusionary criteria were set based on a separate set of pilot data $(\mathrm{N}=13$ collected, $\mathrm{N}=8$ retained for pilot analysis) and were specified in the preregistration. Analyses are based on data from the remaining participants $(\mathrm{N}=20)$.

We subjected memory test manual response accuracy to a 2 (memory load: load-1, load-2) $\times 5$ (trial type: $\mathrm{T}_{\mathrm{M}} \mathrm{D}_{\mathrm{X}}, \mathrm{T}_{\mathrm{N}} \mathrm{D}_{\mathrm{X}}$, $\left.\mathrm{T}_{\mathrm{M}} \mathrm{D}_{\mathrm{N}}, \mathrm{T}_{\mathrm{N}} \mathrm{D}_{\mathrm{N}}, \mathrm{T}_{\mathrm{N}} \mathrm{D}_{\mathrm{M}}\right)$ repeated-measures ANOVA. ${ }^{2}$ There was a significant main effect of memory load $(F(1,19)=$ $163.87, p<.001, \eta_{p}^{2}=.90$ (90\% CI: .79, .93)), with accuracy

\footnotetext{
${ }^{2}$ See Table S1 in the Supplementary Materials for condition means.
}

test matched a color in memory. The memory array squares could be the same color (load-1) or different colors (load-2). The saccade task yielded five different trial types, depending on whether the target or distractor disks matched a color in memory, for each memory load. All trial types were equally likely and randomly intermixed within each block

greater under load-1 $(\mathrm{M}=91.6 \%, \mathrm{SD}=4.6 \%)$ compared to load-2 $\left(\mathrm{M}=79.3 \%, \mathrm{SD}=7.5 \% ; t(19)=12.84, p<.001, \eta_{p}^{2}=\right.$ $.90)$, but no significant main effect of trial type $(F(4,76)=$ $\left.1.60, p=.182, \eta_{p}^{2}=.08\right)$ or interaction $(F(4,76)=2.43, p=$ $\left..055, \eta_{p}^{2}=.11\right)$. Given the dual-task nature of this paradigm and the difficult within-category memory test, reduced performance when two colors were maintained in memory compared to one is not unexpected. Whether the VWMmatching target or distractor disk was an exact or inexact match to a memory array color did not meaningfully interact with memory test performance (see Supplementary Materials for details).

The critical analysis for the current study examined the probability that a saccade was directed to a memorymatching distractor. For this, we examined the trajectory of the first eye movement that occurred after the saccade target and distractor (if present) disks appeared. These saccades were categorized as being directed either toward the target (left/right horizontal meridian $\pm 45^{\circ}$ ) or toward the distractor (upper/lower vertical meridian $\pm 45^{\circ}$ ). As mentioned above, only trials during which the first saccade was directed to a relevant location (up/down/left/right $90^{\circ}$ wedge containing a target or distractor disk) were included for analysis (2.6\% of all trials rejected). Trials with eye movements that were excessively fast $(<90 \mathrm{~ms})$ or slow ( $>600 \mathrm{~ms})(4.9 \%$ of all trials rejected) or that were missing eye-movement data ( $1 \%$ of all trials rejected) were excluded. For all remaining trials $(94.7 \%$ 
of trials retained (some trials met multiple exclusion criteria)), when an initial saccade was directed toward a distractor, the trial was categorized as "capture," whereas if the initial saccade was directed toward the target, the trial was categorized as "no capture." The proportion of "capture" trials was calculated for each distractor-present condition (see Fig. 2).

When multiple representations are being maintained in VWM, there are competing predictions for the likelihood of oculomotor capture by a VWM-matching distractor. If the default state of multiple VWM representations is accessory, we should observe capture on load-1 but not load- 2 trials because the VWM representations will no longer interact with attentional guidance. Alternatively, if the default state of multiple VWM representations is active, we should observe similar rates of capture on load-1 and load-2 trials, as VWM representations will interact with attentional guidance regardless of how many are maintained. Lastly, if the default state of multiple VWM representations is a mixture of active and accessory states such that one item is in an active state at any particular time, we should observe reduced oculomotor capture on load-2 compared to load-1 trials, as the VWMmatching distractor will match the one VWM representation held in an active state on load-2 trials less frequently.

Consistent with the third hypothesis, we observed greater oculomotor capture by a VWM-matching distractor compared to a non-matching distractor both on load-1 (26\% vs. $6 \%$; $\left.t(19)=7.02, p<.001, \eta_{p}^{2}=.72\right)$ and load-2 trials $(12 \%$ vs. $\left.6 \% ; t(19)=5.17, p<.001, \eta_{p}^{2}=.58\right)$. Importantly, capture was reduced for load-2 compared to load-1 $(t(19)=7.13, p<.001$,

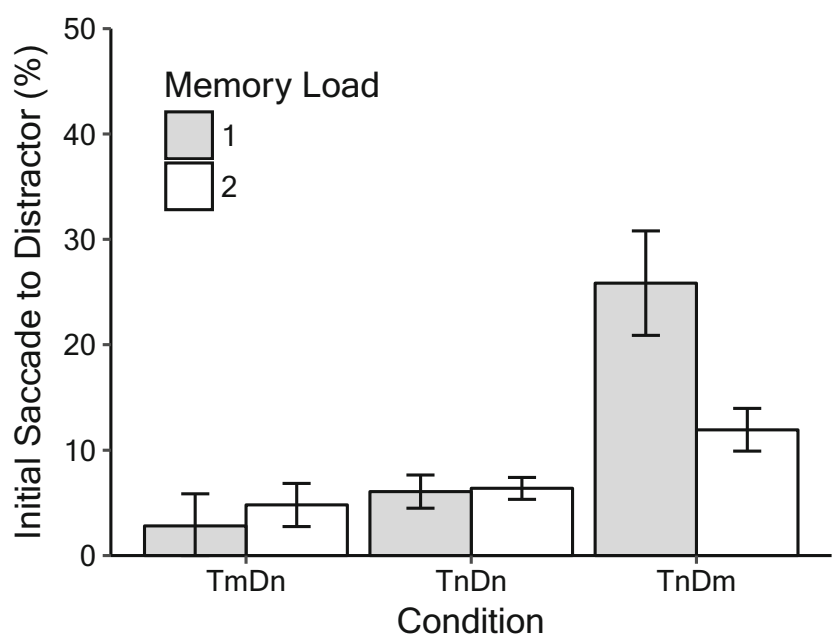

Fig. 2 Initial saccades were more frequently directed to a memorymatching distractor compared to a non-matching distractor both with a one-item $\left(26 \%\right.$ vs. $\left.6 \% ; \mathrm{t}(19)=7.02, \mathrm{p}<.001, \eta_{p}^{2}=.72\right)$ and two-item $\left(12 \%\right.$ vs. $\left.6 \% ; \mathrm{t}(19)=5.17, \mathrm{p}<.001, \eta_{p}^{2}=.58\right)$ memory load. Critically, oculomotor capture under high load was reduced compared to capture under low load (12\% vs. $26 \%$; $\mathrm{t}(19)=7.13, \mathrm{p}<.001, \eta_{p}^{2}=.73$ ), suggesting that multiple colors were held in a mixture of active and accessory states. Error bars indicate \pm within-subject $95 \%$ confidence intervals (Morey, 2008) $\eta_{p}^{2}=.73$ ), suggesting that multiple VWM representations were held in a mixture of active and accessory states. This same pattern of oculomotor capture was present for both exact and inexact VWM-matching distractors (see Supplementary Materials for details).

In fact, the reduction in oculomotor capture from load-1 to load-2 was nearly half. If, when multiple items were being maintained in VWM, one item was randomly assigned to an active state and the other item was assigned to an accessory state, a VWM-matching distractor disk would match the active VWM color half as often as when only a single item is maintained in VWM. To quantify this, we compared the capture rate on load- 2 trials to half the capture rate on load-1 trials and found no difference $(12 \%$ vs. $13 \% ; t(19)=0.76, p=.459$, $\left.\eta_{p}^{2}=.03\right)$. A Bayes Factor analysis indicated that the null was in excess of three times likelier than the alternative $\left(\mathrm{BF}_{01}=\right.$ 3.34). These results suggest that one item at a time is held in an active state under these conditions.

It is worth noting, however, that a small amount of capture was observed for $\mathrm{T}_{\mathrm{N}} \mathrm{D}_{\mathrm{N}}$ trials. To calculate an appropriate baseline, we simulated the amount of "capture" due to an imaginary distractor on $\mathrm{T}_{\mathrm{N}} \mathrm{D}_{\mathrm{X}}$ trials, when no actual distractor was present. The rate of "capture" was less than $1 \%$, on average, and significantly less than when compared to $\mathrm{T}_{\mathrm{N}} \mathrm{D}_{\mathrm{N}}$ trials for both load-1 $\left(0.3 \%\right.$ vs. $5.8 \% ; t(19)=3.10, p=.006, \eta_{p}^{2}=$ $.11)$ and load-2 $\left(0.7 \%\right.$ vs. $6.4 \% ; t(19)=3.04, p=.007, \eta_{p}^{2}=$ .10). Taking the rate of $\mathrm{T}_{\mathrm{N}} \mathrm{D}_{\mathrm{N}}$ capture as a baseline, and removing it from the rate of $\mathrm{T}_{\mathrm{N}} \mathrm{D}_{\mathrm{M}}$ capture, capture under load-1 (19.3\%) is almost four times greater than capture under load-2 (5.2\%). This is consistent with the possibility that memory items are dynamically cycled between active and accessory states. That is, if the distractor and saccade target disks appear when one VWM item is being promoted from an accessory to active state and the other demoted from an active to accessory state, neither of the VWM items are apt to capture attention.

To examine whether differences in saccadic precision across load-1 and load- 2 might explain the observed pattern of attentional capture, we first plotted coordinates of the landing position for the initial saccade on each $\mathrm{T}_{\mathrm{N}} \mathrm{D}_{\mathrm{M}}$ trial relative to the distractor and target positions (see Supplementary Fig. 1). Qualitatively, saccades clustered around distractor and target locations with equal precision across the two memory conditions. To quantify this, we measured the initial saccade precision on capture trials relative to distractor position, and precision on non-capture trials relative to target position, separately for load-1 and load-2 trials. Mean error for capture trials relative to distractor position was $0.97^{\circ}$ visual angle for load-2, and $0.99^{\circ}$ visual angle for load-1, a difference that was not significant $\left(t(15)=0.28, p=.79, \eta_{p}^{2}=.001, \mathrm{BF}_{01}=3.8\right.$; note: four subjects with no capture under load-2 were excluded from this analysis). Mean error for non-capture trials 
relative to target position was $1.13^{\circ}$ visual angle for load-2, and $1.30^{\circ}$ visual angle for load- 1 , a difference that was statistically significant $\left(t(19)=3.18, p=.005, \eta_{p}^{2}=.11\right)$. Notably, however, this difference suggests that saccadic precision was actually improved under load- 2 compared with load- 1 . Thus, we see no evidence that the load-based effect on capture was due to lower saccadic precision under load-2.

One possible explanation for the reduced capture on load-2 trials is that participants were more likely to forget one of the colors on load-2 trials, as indicated by reduced memory test accuracy for load- 2 compared to load- 1 trials, and were therefore less subject to oculomotor capture by a VWM-matching distractor if it matched a color no longer in VWM. However, when we restrict the capture analysis to trials on which participants responded correctly to the memory test, the same pattern emerges (see Supplementary Materials for details). These results suggest that the reduced capture by a VWM-matching distractor in load-2 compared to load-1 cannot be explained simply by reduced memory of the colors maintained in VWM.

A more parsimonious explanation for the current results is that, when multiple items were maintained in VWM, they were held in a mixture of active and accessory states such that only a single item was in an active state at any particular time. It is unclear, however, whether individual items in VWM cycle through active and accessory states during a trial or if one item is preferentially maintained in an active state throughout the trial. However, when adjusted for baseline rates of capture, it would appear that capture is less than $50 \%$ as likely under high compared to low load. This would suggest that items are cycling in and out of the active state, as discussed earlier. Either configuration of active and accessory states within VWM, without the task demands to maintain multiple VWM items in an active state, could begin to resolve previously discrepant findings that sometimes suggested guidance of attention by multiple VWM items, while at other times suggested that none of the items in VWM could influence the guidance of attention when multiple items were held in VWM.

Previous work supporting the latter typically relied on RT measures of capture. In fact, if we instead examine the time-totarget-fixation (analogous to RT) in the current dataset, we again find evidence of capture by a VWM-matching distractor on load-1 trials $\left(\mathrm{M}=96 \mathrm{~ms}\right.$ difference $\left(\mathrm{T}_{\mathrm{N}} \mathrm{D}_{\mathrm{M}}-\mathrm{T}_{\mathrm{N}} \mathrm{D}_{\mathrm{N}}\right) ; t(19)=$ $\left.4.49, p<.001, \eta_{p}^{2}=.52\right)$, but not load-2 $(\mathrm{M}=17 \mathrm{~ms}$ difference $\left.\left(\mathrm{T}_{\mathrm{N}} \mathrm{D}_{\mathrm{M}^{-}} \mathrm{T}_{\mathrm{N}} \mathrm{D}_{\mathrm{N}}\right) ; t(19)=1.51, p=.147, \eta_{p}^{2}=.11\right)$, a similar pattern to the RT results found by van Moorselaar et al. (2014). This discrepancy between oculomotor and time-totarget-fixation results illustrates the value of discrete measures of capture such as the one used here.

\section{Conclusions}

We found clear evidence that maintaining multiple items in VWM reduces, but does not eliminate, attentional capture by memory-matching items. Moreover, the reduction in capture was not significantly different from halving the load-1 capture rate, and was perhaps reduced by more than half, suggesting that time spent in an "active" state was shared across the multiple items in VWM. Though it is possible to maintain multiple VWM representations in an active state that can simultaneously influence attentional guidance, there is evidence that this configuration of attention and VWM systems is less efficient (Menneer, Barrett, Phillips, Donnelly, \& Cave, 2007; Stroud, Menneer, Cave, Donnelly, \& Rayner, 2011) and may be more effortful. To mitigate expending unnecessary effort, multiple representations in VWM may not be held in an active state unless required by current task demands. The saccade portion of the task described here did not rely on the memory items. In the absence of these task demands, the present results support the hypothesis that multiple representations in VWM are held in a mixture of active and accessory states.

Multiple possible causes for the reduction in capture under load-2 remain, but we view it as unlikely that the observed reduction is due to, for instance, interference between memory load and saccadic performance. When we analyzed the precision of saccade landing position, we found that there were no differences between load-1 and load-2 in the degree of 'error' with respect to distractor locations, when capture occurred. Further, non-capture target-directed saccades were actually slightly but significantly more precise under load-2 than load-1. We cannot fully rule out the possibility that reduced capture is due to a less precise representation of two items under load-2. However, we found it notable that similar rates of capture occurred when memory-probe responses were inaccurate as when they were accurate, suggesting that the fidelity of the memory representation played a limited role in determining whether capture occurred under either low or high load.

In the current study, we observed almost exactly a 50\% reduction in capture by memory-matching distractors under load-2 compared with load-1. While we interpreted this as indicating that only one item is held in an 'active' state at a time, the true reduction in capture is likely greater than $50 \%$ : when we factored "baseline" capture by a non-matching distractor into consideration, the reduction in capture from load-1 to load-2 was closer to $75 \%$. A possible explanation for this is that cycling items between active and accessory states in VWM might result in periods during which neither VWM item can interact with attention. If this dynamic cycling between states explains the observed reduction in capture beyond that predicted by the reduced potential for capture due to increased memory load, it suggests that the default configuration within VWM is not that one item is "selected" and held in 
an active state, while the other item is held in an accessory state, for the entire duration of the maintenance period.

We cannot rule out that the precise amount of loaddependent capture observed here might depend on other factors such as target-distractor distance or the predictability of the distractor object location, and thus the precise ratio of capture trials should not be over-interpreted. For instance, although the overall rate of capture was reduced compared to the paradigm used here, varying target-distractor distance has been shown to modulate attentional capture such that a salient distractor closer to the target more frequently elicited oculomotor capture than a salient distractor further away from the target (Gaspelin, Leonard, \& Luck, 2017); we might observe different capture ratios under varying target-distractor distances, though we would predict that the reduced rate of capture due to a load of two items would be proportionally scaled according to the overall capture rate due to location. On the other hand, if participants were simply inhibiting both possible distractor positions on some trials, this type of attentional control and possible mitigation of attentional capture would not be expected to interact with memory load and thus would be an unlikely explanation for the observed reduction in capture from load-1 to load-2. Further examining the precise ratio of load-dependent capture will be important for future work, but the critical finding for the current work is that attentional capture was significantly reduced under load-2 compared to load-1.

The current study takes an important step toward resolving an ongoing debate over the nature of interaction between visual attention and VWM systems. The discrepancy between attentional capture effects observed using oculomotor measures - capture under both low and high VWM loads - and those observed using RT-like measures - time-to-targetfixation revealed capture under low but not high VWM load - suggest a possible explanation for the discrepancy in extant studies. Resolution of this debate over the interface between VWM and attention will have implications for the general architecture of attention and memory systems: some models of working memory suggest that only one item can be held in the "focus of attention" and made available for other processes (McElree, 2001; Oberauer, 2002), whereas others suggest that multiple items can influence other processes (Cowan, 2001). Our results suggest that, in the absence of task demands to maintain multiple VWM items in an active state, a single VWM item is held in an active state and can influence attentional guidance.

Open Practices Statement This study was formally preregistered and can be accessed via the Open Science Framework at: https://osf.io/bns3y/register/565fb3678c5e4a66b5582f67?view_ only $=5 \mathrm{c} 8 \mathrm{~b} 3683232 \mathrm{~b} 4965 \mathrm{a} 755227 \mathrm{e} 1835 \mathrm{~d} 7 \mathrm{af}$

Funding This research was supported by National Science Foundation grants (BCS 1558535 and OIA 1632849) to Timothy J. Vickery.

\section{References}

Bahle, B., Beck, V. M., \& Hollingworth, A. (2018). The architecture of interaction between visual working memory and visual attention. Journal of Experimental Psychology: Human Perception and Performance. https://doi.org/10.1037/xhp0000509

Beck, V. M., \& Hollingworth, A. (2017). Competition in saccade target selection reveals attentional guidance by simultaneously active working memory representations. Journal of Experimental Psychology: Human Perception and Performance, 43(2), 225230. https://doi.org/10.1037/xhp0000306

Beck, V. M., Hollingworth, A., \& Luck, S. J. (2012). Simultaneous Control of Attention by Multiple Working Memory Representations. Psychological Science, 23(8), 887-898. https:// doi.org/10.1177/0956797612439068

Cowan, N. (2001). The magical number 4 in short-term memory: a reconsideration of mental storage capacity. The Behavioral and Brain Sciences, 24(1), 87-185.

Downing, P., \& Dodds, C. (2004). Competition in visual working memory for control of search. Visual Cognition, 11(6), 689-703. https:// doi.org/10.1080/13506280344000446

Gaspelin, N., Leonard, C. J., \& Luck, S. J. (2017). Suppression of overt attentional capture by salient-but-irrelevant color singletons. Attention, Perception, \& Psychophysics, 79(1), 45-62. https://doi. org/10.3758/s13414-016-1209-1

Grubert, A., \& Eimer, M. (2015). Rapid Parallel Attentional Target Selection in Single-Color and Multiple-Color Visual Search. Journal of Experimental Psychology: Human Perception and Performance, 41(1), 86-101. https://doi.org/10.1037/xhp0000019

Grubert, A., \& Eimer, M. (2016). All set, indeed! N2pc components reveal simultaneous attentional control settings for multiple target colors. Journal of Experimental Psychology: Human Perception and Performance, 42(8), 1215-1230. https://doi.org/10.1037/ xhp0000221

Hollingworth, A., \& Beck, V. M. (2016). Memory-based attention capture when multiple items are maintained in visual working memory. Journal of Experimental Psychology: Human Perception and Performance, 42(7), 911-917. https://doi.org/10.1037/xhp0000230

Hollingworth, A., Matsukura, M., \& Luck, S. J. (2013). Visual working memory modulates rapid eye movements to simple onset targets. Psychological Science, 24(5), 790-6. https://doi.org/10.1177/ 0956797612459767

Houtkamp, R., \& Roelfsema, P. R. (2006). The effect of items in working memory on the deployment of attention and the eyes during visual search. Journal of Experimental Psychology: Human Perception and Performance, 32(2), 423-442. https://doi.org/10.1037/00961523.32.2.423

McElree, B. (2001). Working memory and focal attention. Journal of Experimental Psychology: Learning, Memory, and Cognition, 27(3), 817-35.

Menneer, T., Barrett, D. J. K., Phillips, L., Donnelly, N., \& Cave, K. R. (2007). Costs in Searching for Two Targets: Dividing Search Across Target Types Could Improve Airport Security Screening. Applied Cognitive Psychology, 21, 915-932. https://doi.org/10.1002/acp. 1305

Morey, R. D. (2008). Confidence Intervals from Normalized Data: A correction to Cousineau (2005). Tutorial in Quantitative Methods for Psychology, 4(2), 61-64.

Oberauer, K. (2002). Access to information in working memory: Exploring the focus of attention. Journal of Experimental Psychology: Learning, Memory, and Cognition, 28(3), 411-421. https://doi.org/10.1037//0278-7393.28.3.411

Olivers, C. N. L., Meijer, F., \& Theeuwes, J. (2006). Feature-based memory-driven attentional capture: visual working memory content affects visual attention. Journal of Experimental Psychology: Human 
Perception and Performance, 32(5), 1243-1265. https://doi.org/10. 1037/0096-1523.32.5.1243

Olivers, C. N. L., Peters, J., Houtkamp, R., \& Roelfsema, P. R. (2011). Different states in visual working memory: when it guides attention and when it does not. Trends in Cognitive Sciences, 15(7), 327-34. https://doi.org/10.1016/j.tics.2011.05.004

Soto, D., Greene, C. M., Chaudhary, A., \& Rotshtein, P. (2012). Competition in working memory reduces frontal guidance of visual selection. Cerebral Cortex, 22(5), 1159-1169. https://doi.org/10. 1093/cercor/bhr190

Stroud, M. J., Menneer, T., Cave, K. R., Donnelly, N., \& Rayner, K. (2011). Search for multiple targets of different colours: Misguided eye movements reveal a reduction of colour selectivity. Applied Cognitive Psychology, 25(6), 971-982. https://doi.org/10.1002/acp. 1790

van Moorselaar, D., Theeuwes, J., \& Olivers, C. N. L. (2014). In competition for the attentional template: Can multiple items within visual working memory guide attention? Journal of Experimental Psychology: Human Perception and Performance, 40(4), 1450 64. https://doi.org/10.1037/a0036229

Publisher's note Springer Nature remains neutral with regard to jurisdictional claims in published maps and institutional affiliations. 